\title{
PENCILS OF SMALL DEGREE ON CURVES ON UNNODAL ENRIQUES SURFACES
}

\author{
NILS HENRY RASMUSSEN AND SHENGTIAN ZHOU
}

\begin{abstract}
We use vector-bundle techniques in order to compute $\operatorname{dim} W_{d}^{1}(C)$ where $C$ is general and smooth in a linear system on an unnodal Enriques surface. We furthermore find new examples of smooth curves on Enriques surfaces with an infinite number of $g_{\operatorname{gon}(C)}^{1}$ 's.
\end{abstract}

\section{INTRODUCTION}

Let $S$ be a smooth surface over $\mathbb{C}$, and $L$ a line-bundle on $S$. Let $W_{d}^{r}(C)$ be the BrillNoether variety, parametrising complete $g_{d}^{s}$ 's on $C$ for $s \geq r$. We will be concerned with finding the dimension of $W_{d}^{1}(C)$ for small $d$ when $S$ is an unnodal Enriques surface.

The theory on the dimension of $W_{d}^{r}(C)$ dates back to 1874, when Alexander von Brill and Max Noether made an incomplete proof stating that $\operatorname{dim} W_{d}^{r}(C)=\rho(g, r, d):=g-(r+1)(g-$ $d+r)$ provided $C$ is general of genus $g$. It was first much later that strict proofs for this were presented ([KL72], Kem71, GH80]). In 1987, a new proof was constructed by Lazarsfeld ([Laz86]) involving use of vector-bundle techniques for curves on K3 surfaces, exploiting the fact that for any $g \geq 2$, a K3 surface with Picard group $\mathbb{Z} C$ with $C$ a smooth genus $g$ curve can be constructed. These vector-bundle techniques, which were also developed by Tuyring ([Tyu87]), were later used to study the gonality and Clifford index of any smooth curve on an arbitrary K3 surface ([CP95], [Knu03], [Knu09b], AF11]). These methods have also lately been applied in the case of Enriques surfaces and rational surfaces with an anticanonical pencil ([Knu01, [KL09], Knu09a], [LC12]).

The dimension of $W_{d}^{1}(C)$ was studied in [AF11] and [LC12] because of a result by Aprodu in 2005 (Apr05), stating that if $\operatorname{dim} W_{d}^{1}(C)=d-\operatorname{gon}(C)$ for $d \leq g-\operatorname{gon}(C)+2$, then the Green and Green-Lazarsfeld conjectures are satisfied. These conjectures state that the Clifford index and gonality can be read off minimal free resolutions of $\bigoplus_{n} H^{0}\left(C, \mathcal{O}_{C}\left(n K_{C}\right)\right)$ and $\bigoplus_{n} H^{0}\left(C, \mathcal{O}_{C}(n A)\right)$ for $\operatorname{deg}(A) \gg 0$, respectively (see [Gre84] and [GL87]).

In this article, we make an attempt at finding the dimension of $W_{d}^{1}(C)$ when $C$ is a smooth curve on an unnodal Enriques surface $S$. A smooth surface over $\mathbb{C}$ is an Enriques surface if $h^{1}\left(S, \mathcal{O}_{S}\right)=0,2 K_{S} \sim 0$ and $K_{S} \nsim 0$. One defines

$$
\phi(L):=\min \left\{L . E \mid E \in \operatorname{Pic}(S), E^{2}=0, E \not \equiv 0\right\}
$$

and

$$
\mu(L):=\min \left\{L \cdot B-2 \mid B \in \operatorname{Pic}(S) \text { with } B \text { effective, } B^{2}=4, \phi(B)=2 \text {, and } B \not \equiv L\right\} .
$$

1991 Mathematics Subject Classification. 14H51, 14J28, 14J60. 
By [KL09], the generic gonality for smooth curves in $|L|$, which we denote by $k$, is given by

$$
k=\min \left\{2 \phi(L), \mu(L),\left\lfloor\frac{L^{2}}{4}\right\rfloor+2\right\} .
$$

Furthermore, $k=\mu(L)<2 \phi(L)$ precisely when:

- $L^{2}=\phi(L)^{2}$ with $\phi(L) \geq 2$ and even, in which case $k=\mu(L)=2 \phi(L)-2$; or

- $L^{2}=\phi(L)^{2}+\phi(L)-2$ with $\phi(L) \geq 3, L \not \equiv 2 D$ for $D$ such that $D^{2}=10, \phi(D)=3$, in which case $k=2 \phi(L)-1$ for $\phi(L) \geq 5$ and $k=2 \phi(L)-2$ for $\phi(L)=3,4$.

If $\left(L^{2}, \phi(L)\right)=(30,5),(22,4),(20,4),(14,3),(12,3)$ or $(6,2)$, then $k=\left\lfloor\frac{L^{2}}{4}\right\rfloor+2=\phi(L)-1$.

In all other cases, $k=2 \phi(L)$.

Our main result is the following:

Theorem 1.1. Let $S$ be an unnodal Enriques surface, and let $|L|$ be an ample linear system with $L^{2} \geq 2$ such that $k=2 \phi(L)<\mu(L)$. Then, for $k \leq d \leq g-k$ and $C$ general in $|L|$,

$$
\operatorname{dim} W_{d}^{1}(C)=d-k .
$$

Remark 1.2. In the case where $L=n\left(E_{1}+E_{2}\right)$ for $\geq 3$ and $E_{1} \cdot E_{2}=2$, we have $k=$ $\mu(L)<2 \phi(L)$, by [KL09, Corollary 1.5 (a)]. In Example 5.1] we prove that there exists a sub-linear system $\mathfrak{d} \subseteq|L|$ of smooth curves such that for general $C \in \mathfrak{d}$, there exist infinitely many $g_{\text {gon }(C)}^{1}$ 's. These curves are non-exceptional and are, as far as we know, new examples of curves with an infinite number of $g_{\mathrm{gon}(C)}^{1}$ 's.

Remark 1.3. A conjecture by Martens ([Mar84, Statement T, page 280]) states that if $\operatorname{dim} W_{\operatorname{gon}(C)}^{1}(C)=0$, then $\operatorname{dim} W_{d}^{1}(C)=d-\operatorname{gon}(C)$ for $d \leq g-\operatorname{gon}(C)+2$; and that if $\operatorname{dim} W_{\operatorname{gon}(C)}^{1}(C)=1$, then $\operatorname{dim} W_{d}^{1}(C)=d-\operatorname{gon}(C)+1$ for $d \leq g-\operatorname{gon}(C)+2$. We therefore expect that Theorem 1.1 is valid for $d \leq g-k+2$, and hence that the Green and Green-Lazarsfeld conjectures are satisfied for the curves in question.

This paper is organised as follows: In Section 2, we introduce the basic results of BrillNoether theory and the vector-bundles associated to the pairs $(C, A)$, where $|A|$ is a $g_{d}^{1}$ on $C$. In Section 3, we prove Theorem 1.1 in the case where the general vector-bundles are nonstable, while the stable case is covered in Section 4. We close with an example of a sub-linear system of curves with an infinite number of $g_{\mathrm{gon}(C)}^{1}$ 's in Section 5 ,

Acknowledgments. Thanks to Andreas Leopold Knutsen for introducing us to this subject, and for valuable comments and remarks.

\section{Preliminaries}

2.1. Brill-Noether theory. Let $C$ be a smooth curve over $\mathbb{C}$, and let $r$ and $d$ be nonnegative integers. Then there is a variety $W_{d}^{r}(C)$ that parametrises all complete $g_{d}^{s}$, s on $C$, for all $s \geq r$.

Let $|A|$ be a complete $g_{d}^{r}$ on $C$, and let $\mu_{0, A}: H^{0}\left(C, \mathcal{O}_{C}(A)\right) \otimes H^{0}\left(C, \mathcal{O}_{C}\left(K_{C}-A\right)\right) \rightarrow$ $H^{0}\left(C, \mathcal{O}_{C}\left(K_{C}\right)\right)$ be the cup-product mapping. (This is known as the Petri map.) Then, from [ACGH85, IV, Proposition 4.2], we have

$$
\operatorname{dim} T_{[A]} W_{d}^{r}(C)=\rho(g, r, d)+\operatorname{dim} \operatorname{ker}\left(\mu_{0, A}\right),
$$


where $\rho(g, r, d):=g-(r+1)(g-d+1)$ is called the Brill-Noether Number, and also known as "the expected dimension of $W_{d}^{r}(C)$ ".

Furthermore, if $|A|$ is base-point free and $h^{0}\left(C, \mathcal{O}_{C}(A)\right)=2$, then the base-point free pencil trick ([ACGH85, page 126]) gives us

$$
\operatorname{ker} \mu_{0, A}=H^{0}\left(C, \mathcal{O}_{C}\left(K_{C}-2 A\right)\right) .
$$

One defines the gonality of $C$ to be the smallest $d$ such that there exists a $g_{d}^{1}$ on $C$, and denotes it by $\operatorname{gon}(C)$. It is known that for any smooth curve $C$ of genus $g$,

$$
\operatorname{gon}(C) \leq\left\lfloor\frac{g+3}{2}\right\rfloor
$$

For the general curve of genus $g$, we have equality in (3). Note that for curves on Enriques surfaces, since it is known that $\phi(C) \leq \sqrt{C^{2}}=\sqrt{2 g-2}$, the gonality is usually not maximal.

Let $W$ be a component of $W_{d}^{1}(C)$ containing $A$. Then,

$$
\text { if } \operatorname{dim} \operatorname{ker} \mu_{0, A}=0 \text { and } d \leq g-\operatorname{gon}(C)+2 \text {, then } \operatorname{dim} W \leq d-\operatorname{gon}(C) \text {. }
$$

Also, note that if the general $g_{d}^{1}$ in $W$ has base-points, then we can obtain these $g_{d}^{1}$ 's by considering $g_{d-1}^{1}$ 's and add base-points. It follows that

(5) if the general $g_{d}^{1}$ 's in $W$ have base-points, then $\operatorname{dim} W \leq \operatorname{dim} W_{d-1}^{1}(C)+1$.

The following definition, which was introduced in [Mar68], generalises the notion of gonality for a curve $C$ :

Definition 2.1. Let $C$ be a smooth curve of genus $g \geq 4$. The Clifford index of $C$ is defined to be

Cliff $(C):=\min \left\{\operatorname{deg}(A)-2\left(h^{0}\left(C, \mathcal{O}_{C}(A)\right)-1\right) \mid h^{0}\left(C, \mathcal{O}_{C}(A)\right) \geq 2\right.$ and $\left.h^{1}\left(C, \mathcal{O}_{C}(A)\right) \geq 2\right\}$.

If $A$ is a divisor on $C$ satisfying $h^{0}\left(C, \mathcal{O}_{C}(A)\right) \geq 2$ and $h^{1}\left(C, \mathcal{O}_{C}(A)\right) \geq 2$, then one says that $A$ contributes to the Clifford index of $C$, and $A$ is then defined to have Clifford index $\operatorname{Cliff}(A):=\operatorname{deg}(A)-2\left(h^{0}\left(C, \mathcal{O}_{C}(A)\right)-1\right)$.

If $C$ is hyperelliptic of genus 2 or 3 , one defines $\operatorname{Cliff}(C)=0$; and if $C$ is non-hyperelliptic of genus 3 , one defines $\operatorname{Cliff}(C)=1$.

It was proved in [CM91, Theorem 2.3] that $\operatorname{Cliff}(C) \in\{k-2, k-3\}$, where $k=\operatorname{gon}(C)$. We have Cliff $(C)=k-2=\left\lfloor\frac{g-1}{2}\right\rfloor$ if $C$ is general in $\mathscr{M}_{g}$ for $g \geq 2$. If $\operatorname{Cliff}(C)=k-3$, then $C$ is said to be exceptional.

2.2. Vector-bundle techniques. Let $S$ be an Enriques surface, and let $L$ be a line-bundle on $S$. One defines $\mathcal{W}_{d}^{1}|L|:=\left\{\left.(C, A)|C \in| L\right|_{s}, A \in W_{d}^{1}(C)\right\}$, and $\pi: \mathcal{W}_{d}^{1}|L| \rightarrow|L|_{s}$ the natural projection map, where $|L|_{s}$ denotes the smooth curves of $|L|$. Each fibre of $\pi$ is isomorphic to $W_{d}^{1}(C)$.

Let $\mathcal{W}$ be an irreducible component of $\mathcal{W}_{d}^{1}|L|$ such that $\pi$ restricted to $\mathcal{W}$ dominates. By (5)), we can assume that for general $(C, A)$ in $\mathcal{W},|A|$ is base-point free. It thus makes sense to study the associated Lazarsfeld-Mukai vector bundles, $\mathcal{F}_{C, A}$ and $\mathcal{E}_{C, A}$ (see [Laz86]).

Let $A \in W_{d}^{1}(C) \backslash W_{d}^{2}(C)$ be base-point free. The vector-bundle $\mathcal{F}_{C, A}$ is defined by

$$
0 \longrightarrow \mathcal{F}_{C, A} \longrightarrow H^{0}\left(S, \mathcal{O}_{S}(A)\right) \otimes \mathcal{O}_{S} \stackrel{\text { ev }}{\longrightarrow} \mathcal{O}_{S}(A) \longrightarrow 0 .
$$


One denotes the dual of $\mathcal{F}$ by $\mathcal{F}^{\vee}=\mathcal{E}_{C, A}$. Dualising (6), one gets

$$
0 \rightarrow H^{0}\left(S, \mathcal{O}_{S}(A)\right)^{\vee} \otimes \mathcal{O}_{S} \rightarrow \mathcal{E}_{C, A} \rightarrow \mathcal{O}_{C}\left(K_{C}-A+\left.K_{S}\right|_{C}\right) \rightarrow 0 .
$$

Note that because we are assuming $d \leq g-\operatorname{gon}(C)$, then $h^{0}\left(C, \mathcal{O}_{C}\left(K_{C}-A+\left.K_{S}\right|_{C}\right)\right)>0$, by Riemann-Roch. Hence, the vector-bundles $\mathcal{E}_{C, A}$ are globally generated away from a finite set of points, those points being the possible base-points of $\mathcal{O}_{C}\left(K_{C}-A+\left.K_{S}\right|_{C}\right)$. One has the following properties of $\mathcal{E}_{C, A}$ :

$$
\begin{array}{ll}
\text { - } & c_{1}\left(\mathcal{E}_{C, A}\right)=L \\
\text { - } & c_{2}\left(\mathcal{E}_{C, A}\right)=d \\
\text { - } & h^{0}\left(S, \mathcal{E}_{C, A}^{\vee}\right)=h^{1}\left(S, \mathcal{E}_{C, A}^{\vee}\right)=0, h^{2}\left(S, \mathcal{E}_{C, A}\right)=0 \\
\text { - } & h^{1}\left(S, \mathcal{E}_{C, A}\right)=h^{0}\left(C, \mathcal{O}_{C}\left(A+\left.K_{S}\right|_{C}\right)\right)
\end{array}
$$

Given a vector-bundle $\mathcal{E}$ of $\operatorname{rank} 2$, with $c_{1}(\mathcal{E})=L, c_{2}(\mathcal{E})=d$, and $h^{2}(S, \mathcal{E})=0$, and which is finitely generated away from a finite set of points, then given a two-dimensional subspace $\Lambda$ in $H^{0}(S, \mathcal{E})$, the cokernel of $\Lambda \otimes \mathcal{O}_{S} \hookrightarrow \mathcal{E}$ is isomorphic to $\mathcal{O}_{C_{\Lambda}}(B)$ for some $C_{\Lambda} \in|L|$, and where $B$ is a torsion-free sheaf of rank 1 on $C_{\Lambda}$. If $C_{\Lambda}$ is smooth, then $B \cong \mathcal{O}_{C_{\Lambda}}\left(K_{C_{\Lambda}}-A_{\Lambda}+\left.K_{S}\right|_{C_{\Lambda}}\right)$ for some $|A| \in W_{d}^{1}\left(C_{\Lambda}\right)$, giving us an exact sequence

$$
0 \rightarrow \Lambda \otimes \mathcal{O}_{S} \rightarrow \mathcal{E} \rightarrow \mathcal{O}_{C_{\Lambda}}\left(K_{C_{\Lambda}}-A_{\Lambda}+\left.K_{S}\right|_{C_{\Lambda}}\right) \rightarrow 0
$$

An important tool for us will be the following:

Proposition 2.2. Suppose that $\mathcal{W}$ is a component of $\mathcal{W}_{d}^{1}|L|$ such that $\pi: \mathcal{W} \rightarrow|L|$ dominates. Let $(C, A)$ be sufficiently general in $\mathcal{W}$, and suppose that $|A|$ is base-point free for these $A$. Then there exists an exact sequence

$$
0 \rightarrow H^{0}\left(C,\left.K_{S}\right|_{C}\right) \rightarrow H^{0}\left(C, \mathcal{E}_{C, A}^{\vee} \otimes \mathcal{O}_{C}\left(K_{C}-A\right)\right) \rightarrow H^{0}\left(C, \mathcal{O}_{C}\left(K_{C}-2 A\right)\right) \rightarrow 0 .
$$

In particular, $h^{0}\left(C, \mathcal{E}_{C, A}^{\vee} \otimes \mathcal{O}_{C}\left(K_{C}-A\right)\right)=\operatorname{dim} \operatorname{ker} \mu_{0, A}$.

Proof. We follow the proof of [Par95, Theorem 2]. (See also [LC12, Proposition 3.2].)

Since $|A|$ is base-point free and $h^{0}\left(C, \mathcal{O}_{C}(A)\right)=2$, we have an exact sequence

$$
0 \longrightarrow \mathcal{O}_{C}(-A) \longrightarrow H^{0}\left(C, \mathcal{O}_{C}(A)\right) \otimes \mathcal{O}_{C} \stackrel{\text { ev }}{\longrightarrow} \mathcal{O}_{C}(A) \longrightarrow 0,
$$

where ev is the evalutation morphism.

The diagram

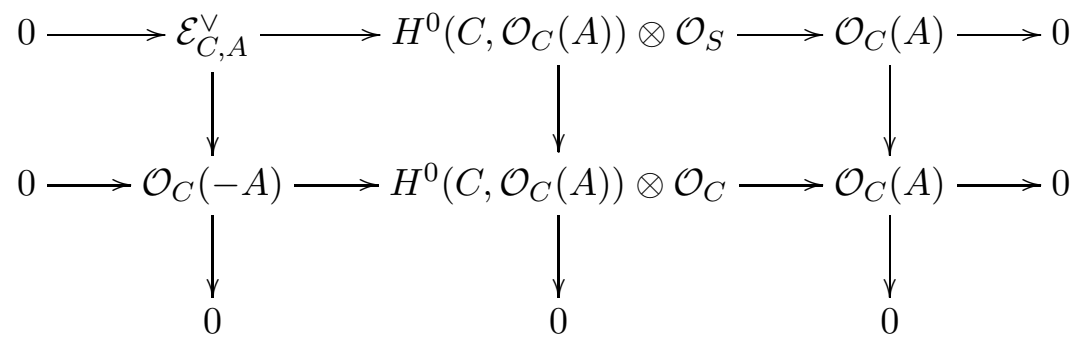

yields a surjection $\left.\mathcal{E}_{C, A}^{\vee}\right|_{C} \rightarrow \mathcal{O}_{C}(-A) \rightarrow 0$, and since $\left.\bigwedge^{2} \mathcal{E}_{C, A}^{\vee}\right|_{C}=\mathcal{O}_{C}\left(-K_{C}+\left.K_{S}\right|_{C}\right)$, the kernel must be $\mathcal{O}_{C}\left(A-K_{C}+\left.K_{S}\right|_{C}\right)$, and we get the sequence

$$
\left.0 \rightarrow \mathcal{O}_{C}\left(A-K_{C}+\left.K_{S}\right|_{C}\right) \rightarrow \mathcal{E}_{C, A}^{\vee}\right|_{C} \rightarrow \mathcal{O}_{C}(-A) \rightarrow 0 .
$$


We tensor with $\mathcal{O}_{C}\left(K_{C}-A\right)$ and get

$$
\left.0 \rightarrow \mathcal{O}_{C}\left(\left.K_{S}\right|_{C}\right) \rightarrow \mathcal{E}_{C, A}^{\vee}\right|_{C} \otimes \mathcal{O}_{C}\left(K_{C}-A\right) \rightarrow \mathcal{O}_{C}\left(K_{C}-2 A\right) \rightarrow 0 .
$$

Taking global sections gives us

$$
\begin{array}{r}
0 \rightarrow H^{0}\left(C, \mathcal{O}_{C}\left(\left.K_{S}\right|_{C}\right)\right) \rightarrow H^{0}\left(C,\left.\mathcal{E}_{C, A}^{\vee}\right|_{C} \otimes \mathcal{O}_{C}\left(K_{C}-A\right)\right) \rightarrow H^{0}\left(C, \mathcal{O}_{C}\left(K_{C}-2 A\right)\right) \\
\rightarrow H^{1}\left(C, \mathcal{O}_{C}\left(\left.K_{S}\right|_{C}\right)\right) .
\end{array}
$$

Note that from (2) we have $H^{0}\left(C, \mathcal{O}_{C}\left(K_{C}-2 A\right)\right)=\operatorname{ker} \mu_{0, A}$. Following an argument identical to [Par95, Lemma 1], we have that the coboundary-map $H^{0}\left(C, \mathcal{O}_{C}\left(K_{C}-2 A\right)\right) \rightarrow$ $H^{1}\left(C, \mathcal{O}_{C}\left(\left.K_{S}\right|_{C}\right)\right)$ up to constant factors is equal to the map $\mu_{1, A, S}: \operatorname{ker}_{0, A} \rightarrow H^{1}\left(C, \mathcal{O}_{C}\left(\left.K_{S}\right|_{C}\right)\right)$ which is given as follows:

The map $\mu_{1, A, S}$ is the composition of the Gaussian map $\mu_{1, A}: H^{0}\left(C, \mathcal{O}_{C}\left(K_{C}-2 A\right)\right) \rightarrow$ $H^{0}\left(C, \mathcal{O}_{C}\left(2 K_{C}\right)\right)$ with the transpose of the Kodaira-Spencer map $\delta_{C, S}^{\vee}: H^{0}\left(C, \mathcal{O}_{C}\left(2 K_{C}\right)\right) \rightarrow$ $\left(T_{C}|L|\right)^{\vee}=H^{1}\left(C, N_{C \mid S}^{\vee} \otimes \mathcal{O}_{C}\left(K_{C}\right)\right)=H^{1}\left(C, \mathcal{O}_{C}\left(\left.K_{S}\right|_{C}\right)\right)$.

The lemma follows from considering a commutative diagram

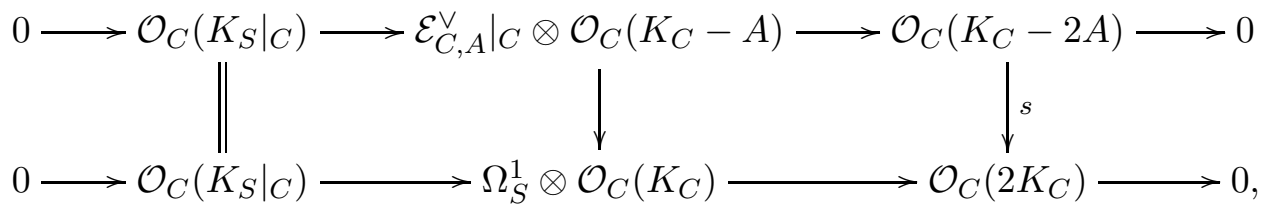

where $\mu_{1, A}$ is found by considering $s$ on the global sections level, and $\delta_{C, S}^{\vee}$ is the coboundary $\operatorname{map} H^{0}\left(C, \mathcal{O}_{C}\left(2 K_{C}\right)\right) \rightarrow H^{1}\left(C, \mathcal{O}_{C}\left(\left.K_{S}\right|_{C}\right)\right)$.

In [Par95, page 197], it is argued that

$$
\operatorname{Im}\left(\mathrm{d} \pi_{C, A}\right) \subset \operatorname{Ann}\left(\operatorname{Im}\left(\mu_{1, A, S}\right)\right) .
$$

We also have a natural inclusion

$$
\operatorname{Ann}\left(\operatorname{Im}\left(\mu_{1, A, S}\right)\right) \subset H^{1}\left(C, \mathcal{O}_{C}\left(\left.K_{S}\right|_{C}\right)\right)^{\vee},
$$

and the latter has dimension $g-1$.

Since by assumption $\pi$ dominates $|L|$, then by Sard's lemma, $\mathrm{d} \pi_{C, A}$ is surjective for general $(C, A)$, and so $\operatorname{Im}\left(\mathrm{d} \pi_{C, A}\right)$ also has dimension $g-1$.

It follows that $\operatorname{Ann}\left(\operatorname{Im}\left(\mu_{1, A, S}\right)\right)=H^{1}\left(C, \mathcal{O}_{C}\left(\left.K_{S}\right|_{C}\right)\right)^{\vee}$, and so $\operatorname{Im}\left(\mu_{1, A, S}\right)=0$. Hence, the sequence

$$
0 \rightarrow H^{0}\left(C, \mathcal{O}_{C}\left(\left.K_{S}\right|_{C}\right)\right) \rightarrow H^{0}\left(C,\left.\mathcal{F}_{C, A}\right|_{C} \otimes \mathcal{O}_{C}\left(K_{C}-A\right)\right) \rightarrow H^{0}\left(C, \mathcal{O}_{C}\left(K_{C}-2 A\right)\right) \rightarrow 0
$$

is exact.

We will prove the main theorem by considering the case where the general $\mathcal{E}_{C, A^{\prime}}$ 's are $\mu_{L^{-}}$ stable and non- $\mu_{L}$-stable.

Definition 2.3. Given a line-bundle $L$ on a surface $S$, a vector-bundle $\mathcal{E}$ is said to be $\mu_{L^{-}}$ stable if for any sub-vector bundle $\mathcal{E}^{\prime}$ of rank $0<\operatorname{rk}\left(\mathcal{E}^{\prime}\right)<\operatorname{rk}(\mathcal{E})$, we have

$$
\frac{c_{1}\left(\mathcal{E}^{\prime}\right) \cdot L}{\operatorname{rk}\left(\mathcal{E}^{\prime}\right)}<\frac{c_{1}(\mathcal{E}) \cdot L}{\operatorname{rk}(\mathcal{E})}
$$




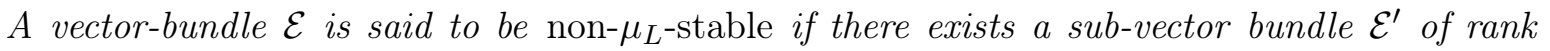
$0<\operatorname{rk}\left(\mathcal{E}^{\prime}\right)<\operatorname{rk}(\mathcal{E})$ satisfying

$$
\frac{c_{1}\left(\mathcal{E}^{\prime}\right) \cdot L}{\operatorname{rk}\left(\mathcal{E}^{\prime}\right)} \geq \frac{c_{1}(\mathcal{E}) \cdot L}{\operatorname{rk}(\mathcal{E})}
$$

2.3. Assumptions. Throughout the article, we will be using the following assumptions:

- $\operatorname{dim} W_{d-1}^{1}(C)=d-1-k$ for $C$ general in $|L|$ (by induction).

- The general $g_{d}^{1}$ 's are base-point free for general $(C, A)$ in a component $\mathcal{W}$ of $\mathcal{W}_{d}^{1}|L|$.

- $k \geq 3$ (since linear growth is always satisfied for hyperelliptic curves. This implies that $\left.L^{2} \geq 4\right)$

- $k \leq d \leq g-k$. In particular, $k \leq \frac{g}{2}$, since Theorem 1.1 is otherwise trivially satisfied

- $\mathcal{W}$ is a component of $\mathcal{W}_{d}^{1}|L|$ such that $\pi: \mathcal{W} \rightarrow|L|$ dominates and for general $C \in|L|$ the fibre over $C$ has dimension $\operatorname{dim} W_{d}^{1}(C)$

\section{The CASE Where the $\mathcal{E}_{C, A}$ 'S ARE NON- $\mu_{L}$-STABLE}

In this section, we will assume that for general $(C, A) \in \mathcal{W}$, the vector-bundles $\mathcal{E}_{C, A}$ are non- $\mu_{L}$-stable. The main result of this section is Proposition 3.9, where we do a parameter count of all possible non- $\mu_{L}$-stable vector-bundles that satisfy the properties of $\mathcal{E}_{C, A}$.

We start by recalling two results, one from [KL07] and one from [KL09], which we will be using several times throughout this section:

Theorem 3.1 ([KL07, Theorem], case of Enriques surfaces). Let $S$ be an Enriques surface, and $\mathcal{O}_{S}(D)$ a line-bundle on $S$ such that $D>0$ and $D^{2} \geq 0$. Then $H^{1}\left(S, \mathcal{O}_{S}(D)\right) \neq 0$ if and only if one of the three following occurs:

(i) $D \sim n E$ for $E>0$ nef and primitive with $E^{2}=0, n \geq 2$ and $h^{1}\left(S, \mathcal{O}_{S}(D)\right)=\left\lfloor\frac{n}{2}\right\rfloor$;

(ii) $D \sim n E+K_{S}$ for $E>0$ nef and primitive with $E^{2}=0, n \geq 3$ and $h^{1}\left(S, \mathcal{O}_{S}(D)\right)=$ $\left\lfloor\frac{n-1}{2}\right\rfloor$

(iii) there is a divisor $\Delta>0$ such that $\Delta^{2}=-2$ and $\Delta . D \leq-2$.

Note that since the Enriques surfaces in question in our article are assumed to be unnodal, then part (iii) of Theorem 3.1 cannot occur.

Lemma 3.2 ([KL09, Lemma 2.12]). Let $L>0$ be a line bundle on an Enriques surface $S$ with $L^{2} \geq 0$. Then there is an integer $n$ such that $1 \leq n \leq 10$ and, for any $i=1, \ldots, n$, there are primitive divisors $E_{i}>0$ with $E_{i}^{2}=0$ and integers $a_{i}>0$ such that

$$
L \equiv a_{1} E_{1}+\cdots+a_{n} E_{n}
$$

and one of the following intesection sets occurs:

(i) $E_{i} \cdot E_{j}=1$ for $1 \leq i<j \leq n$.

(ii) $n \geq 2, E_{1} \cdot E_{2}=2$ and $E_{i} \cdot E_{j}=1$ for $2 \leq i<j \leq n$ and for $i=1,3 \leq j \leq n$.

(iii) $n \geq 3, E_{1} \cdot E_{2}=E_{1} \cdot E_{3}=2$ and $E_{i} . E_{j}=1$ for $3 \leq i<j \leq n$, for $i=1,4 \leq j \leq n$ and for $i=2,3 \leq j \leq n$. 
The following proposition is crucial to our result. The fact that we can assume that the vector-bundles are contained in a short-exact sequence as in (18), where M.L $\geq N . L$, will eventually ensure that the dimensions of extensions of various $\mathcal{O}_{S}(M)$ and $\mathcal{O}_{S}(N) \otimes \mathcal{I}_{\xi}$ is small enough to give us the desired result (see Lemma 3.7).

Proposition 3.3. Suppose $\mathcal{E}_{C, A}$ is non- $\mu_{L}$-stable. Then there exist line-bundles $\mathcal{O}_{S}(M)$ and $\mathcal{O}_{S}(N)$, and a 0 -dimensional subscheme $\xi$, such that $\mathcal{E}_{C, A}$ sits inside an exact sequence

$$
0 \rightarrow \mathcal{O}_{S}(M) \rightarrow \mathcal{E}_{C, A} \rightarrow \mathcal{O}_{S}(N) \otimes \mathcal{I}_{\xi} \rightarrow 0,
$$

satisfying the following conditions:

(a) We have $M+N \sim C$, length $(\xi)=d-M . N$, and $|N|$ is non-trivial and base-component free (implying that $h^{0}\left(S, \mathcal{O}_{S}(N)\right) \geq 2$ ). Furthermore, $h^{2}\left(S, \mathcal{O}_{S}(M-N)\right)=0$ and $h^{0}\left(S, \mathcal{O}_{S}(N-M)\right)=0$, unless $M \sim N+K_{S}$ or $M \sim N$, respectively.

(b) We have $h^{2}\left(S, \mathcal{O}_{S}(M)\right)=0$ and $h^{0}\left(S, \mathcal{O}_{S}(M)\right) \geq 2$.

(c) We have $h^{1}\left(S, \mathcal{O}_{S}(M)\right)=0$.

(d) We have $\left.N\right|_{C} \geq A$.

(e) If $\xi \neq \emptyset$, then $\bar{h}^{1}\left(S, \mathcal{O}_{S}(N)\right)=0$ and $N^{2}>0$.

Note that the points where $\mathcal{E}_{C, A}$ is not globally generated lie along the curve $C$.

Proof. Since $\mathcal{E}_{C, A}$ by assumption is non- $\mu_{L}$-stable, there exists a line-bundle $\mathcal{O}_{S}(M)$ of slope $\geq g-1$ on $C$ that injects into $\mathcal{E}_{C, A}$. We can assume that the injection is saturated, and so we obtain the sequence (18). Note that since $M . C \geq g-1$, then $N . C \leq g-1$.

We have $M+N \sim C$, length $(\xi)=d-M . N$ and $|N|$ base-point free because of (8) and (9), and the fact that $\mathcal{E}_{C, A}$ is gobally generated away from a finite set of points. We have $N$ non-trivial because otherwise, $M . N=0$, implying that $\xi \neq \emptyset$, and this would imply that $h^{0}\left(S, \mathcal{O}_{S}(N) \otimes \mathcal{I}_{\xi}\right)=0$, which contradicts $\mathcal{E}_{C, A}$ being globally generated away from a finite set.

We have $h^{2}\left(S, \mathcal{O}_{S}(M-N)\right)=0$ and $h^{0}\left(S, \mathcal{O}_{S}(N-M)\right)=0$ by the Nakai-Moishezon criterion, using that $L$ by assumption is ample, and that M.L $\geq N . L$.

We now show part (b). Since $M . C \geq g-1$, then since $L$ is ample, $-M+K_{S}$ cannot be effective. It follows that $h^{0}\left(S, \mathcal{O}_{S}\left(-M+K_{S}\right)\right)=0$, and this equals $h^{2}\left(S, \mathcal{O}_{S}(M)\right)$ by Serre duality.

To prove that $h^{0}\left(S, \mathcal{O}_{S}(M)\right) \geq 2$, by part (a), we have $M . N \leq d \leq g-k<g-1$, and this gives us $g-1 \leq M . C=M^{2}+M . N<M^{2}+g-1$, yielding $M^{2}>0$. The result now follows from Riemann-Roch.

Part (c) follows from the fact that $M^{2}>0$ (proven in part (b)) together with Theorem 3.1.

To prove (d), note that by tensoring (18) with $\mathcal{O}_{S}(-M)$ and taking global sections, we get $h^{0}\left(S, \mathcal{E}_{C, A} \otimes \mathcal{O}_{S}(-M)\right) \geq 1$. Rewrite (7) as

$$
0 \rightarrow \mathcal{O}_{S}^{\oplus 2} \rightarrow \mathcal{E}_{C, A} \rightarrow \mathcal{O}_{C}\left(\left.C\right|_{C}-A\right) \rightarrow 0,
$$

tensor with $\mathcal{O}_{S}(-M)$ and take global sections. This gives us an injection $H^{0}\left(S, \mathcal{E}_{C, A} \otimes\right.$ $\left.\mathcal{O}_{S}(-M)\right) \hookrightarrow H^{0}\left(C, \mathcal{O}_{C}\left(\left.N\right|_{C}-A\right)\right)$, proving that $\left.N\right|_{C}-A \geq 0$.

As for (e), suppose that $h^{1}\left(S, \mathcal{O}_{S}(N)\right)>0$. By Theorem 3.1, it follows that $N^{2}=0$. From (d), we then have $d \leq N . C=N .(M+N)=M . N$, contradicting part (a), which states that $d=M \cdot N+\operatorname{length}(\xi)$.

The following lemma gives us an upper bound on $h^{0}(S, \mathcal{E})$, because of $(\underline{18})$. 
Lemma 3.4. Suppose that for general $(C, A) \in \mathcal{W}$, the associated vector-bundle $\mathcal{E}_{C, A}$ is non$\mu_{L}$-stable, so that we have a short-exact sequence as in Proposition 3.3 where $M$ and $N$ are fixed. Then, for general $(C, A)$, we have $h^{1}\left(S, \mathcal{E}_{C, A}\right) \leq 2$ and $h^{1}\left(S, \mathcal{O}_{S}(N) \otimes \mathcal{I}_{\xi}\right) \leq 2$.

Proof. Note that from (17), we are assuming that $\pi: \mathcal{W} \rightarrow|L|$ dominates, and that for general $C \in|L|$, the fibre over $C$ has dimension $W_{d}^{1}(C)$.

Suppose $h^{1}\left(S, \mathcal{O}_{S}(N) \otimes \mathcal{I}_{\xi}\right) \geq 3$. Then taking cohomology of (18), we get a surjection $H^{1}\left(S, \mathcal{E}_{C, A}\right) \rightarrow H^{1}\left(S, \mathcal{O}_{S}(N) \otimes \mathcal{I}_{\xi}\right) \rightarrow 0$, implying that $h^{1}\left(S, \mathcal{E}_{C, A}\right) \geq 3$.

By (11), $h^{1}\left(S, \mathcal{E}_{C, A}\right)=h^{0}\left(C, \mathcal{O}_{C}\left(A+\left.K_{S}\right|_{C}\right)\right)$, giving us $W_{d}^{1}(C)$ dimensions of $g_{d}^{2}$ 's, which is impossible.

The following lemma is necessary for the proof of Proposition 3.6, where we prove that $M . N \geq k-1$. This lemma is (in the Enriques surface case) an improvement of a similar result in AF11, where it is shown that $\left.M\right|_{C}$ contributes to the Clifford index. By using $\left.M\right|_{C}$ instead of $\left.(M+E)\right|_{C}$ in Proposition 3.6, we would only obtain $M . N \geq k-2$.

Lemma 3.5. Suppose we have a sequence as in Proposition 3.3 with $(M-N)^{2} \geq 0$. If there exists a primitive elliptic curve $E$ such that $(M-N) . E>0$ and $h^{0}\left(S, \mathcal{O}_{S}\left(N-E+K_{S}\right)\right) \geq 2$, then $\left.(M+E)\right|_{C}$ contributes to $\operatorname{Cliff}(C)$.

Proof. By (15), we have $k \geq 3$, and so there exist line-bundles on $C$ that contribute to Cliff $(C)$.

We show that $h^{i}\left(C,\left.\mathcal{O}_{S}(M+E)\right|_{C}\right) \geq 2$ for $i=0,1$.

Consider the exact sequence

$$
0 \rightarrow \mathcal{O}_{S}(-C) \rightarrow \mathcal{O}_{S} \rightarrow \mathcal{O}_{C} \rightarrow 0
$$

tensored with $\mathcal{O}_{S}(M+E)$, giving us

$$
\left.0 \rightarrow \mathcal{O}_{S}(-N+E) \rightarrow \mathcal{O}_{S}(M+E) \rightarrow \mathcal{O}_{S}(M+E)\right|_{C} \rightarrow 0 .
$$

Because $h^{0}\left(S, \mathcal{O}_{S}(N)\right) \geq 2$ by Proposition 3.3 , we must have $h^{0}\left(S, \mathcal{O}_{S}(-N+E)\right)=0$. By the same proposition, it follows that $h^{0}\left(S, \mathcal{O}_{S}(M+E)\right) \geq 2$, and so also $h^{0}\left(C,\left.\mathcal{O}_{S}(M+E)\right|_{C}\right) \geq 2$, as desired.

We have $h^{1}\left(C,\left.\mathcal{O}_{S}(M+E)\right|_{C}\right)=h^{0}\left(C, \mathcal{O}_{C}\left(K_{C}-\left.M\right|_{C}-\left.E\right|_{C}\right)\right)=h^{0}\left(C, \mathcal{O}_{S}\left(C+K_{S}-\right.\right.$ $\left.M-E)\left.\right|_{C}\right)=h^{0}\left(C,\left.\mathcal{O}_{S}\left(N-E+K_{S}\right)\right|_{C}\right)$. By considering the sequence (19) tensored with $\mathcal{O}_{S}\left(N-E+K_{S}\right)$, we get

$$
\left.0 \rightarrow \mathcal{O}_{S}\left(-M-E+K_{S}\right) \rightarrow \mathcal{O}_{S}\left(N-E+K_{S}\right) \rightarrow \mathcal{O}_{S}\left(N-E+K_{S}\right)\right|_{C} \rightarrow 0 .
$$

Since $h^{0}\left(S, \mathcal{O}_{S}(M)\right) \geq 2$ by Proposition $3.3, h^{0}\left(S, \mathcal{O}_{S}\left(-M-E+K_{S}\right)\right)=0$, and so $H^{0}\left(S, \mathcal{O}_{S}(N-\right.$ $\left.\left.E+K_{S}\right)\right) \hookrightarrow H^{0}\left(C,\left.\mathcal{O}_{S}\left(N-E+K_{S}\right)\right|_{C}\right)$. We have $h^{0}\left(S, \mathcal{O}_{S}\left(N-E+K_{S}\right)\right) \geq 2$ by assumption, and so $h^{0}\left(C,\left.\mathcal{O}_{S}\left(N-E+K_{S}\right)\right|_{C}\right) \geq 2$ as well.

In the following proposition, we obtain a connection between $M . N$ and the generic gonality in $|L|$. This is used when we make the parameter count of extensions of $\mathcal{O}_{S}(M)$ and $\mathcal{O}_{S}(N) \otimes \mathcal{I}_{\xi}$ in the proof of Proposition 3.9.

Here we use that the general curves in $|L|$ are non-exceptional. This is a consequence of [KL13, Corollary 1.2], where we find that the Clifford index for the general curve in $|L|$ is $k-2$.

Note that Proposition 3.6, in the case of $N^{2}=4$, is the only place where we use the assumption that $\mu(L)>2 \phi(L)$.

Proposition 3.6. Suppose that for general $(C, A)$ in $\mathcal{W}$, the vector-bundle $\mathcal{E}_{C, A}$ is non- $\mu_{L^{-}}$ stable. Suppose furthermore that $\mu(L)>2 \phi(L)=k$. Then $M . N \geq k-1$. 
Proof. Suppose first that $(M-N)^{2} \leq 0$. Then $2 M . N \geq M^{2}+N^{2}$, and so $2 g-2=(M+N)^{2}=$ $M^{2}+2 M . N+N^{2} \leq 4 M . N$, yielding $M . N \geq \frac{g-1}{2}$. Since $k \leq \frac{g}{2}$ by assumption (16), the result follows.

Now suppose $(M-N)^{2}>0$.

We start by considering three special cases, namely $N^{2}=0, N^{2}=2$, and $N^{2}=4$ with $\phi(N)=2$.

Special case 1. If $N^{2}=0$, then we know from Proposition 3.3 that $d \leq N . C=N .(M+$ $N)=M . N$, and so it follows in particular that $k-1 \leq M . N$.

Special case 2. If $N^{2}=2$, note that by Theorem [3.1. $h^{1}\left(S, \mathcal{O}_{S}(N)\right)=0$, so that $h^{0}\left(S, \mathcal{O}_{S}(N)\right)=\frac{1}{2} N^{2}+1=2$. Since $\mathcal{E}_{C, A}$ is globally generated away from a finite set of points, then so must $\left|\mathcal{O}_{S}(N) \otimes \mathcal{I}_{\xi}\right|$, and so all points of $\xi$ must be along base-points of $|N|$ (or else $\operatorname{dim}\left|\mathcal{O}_{S}(N) \otimes \mathcal{I}_{\xi}\right| \leq 0$ ). Since $\mathcal{E}_{C, A}$ is globally generated outside of $C$, this implies that the base-points of $|N|$, and hence also the points of $\xi$, must lie along $C$. However, $h^{1}\left(S, \mathcal{O}_{S}(N) \otimes \mathcal{I}_{\xi}\right)$ indicates (in this particular case) how many points of $\xi$ that lie along base-points of $|N|$, and by Lemma 3.4, it follows that length $(\xi) \leq 2$.

If $d>k$, then this yields $2 \geq$ length $(\xi)=d-M . N>k-M . N$, which leads to $M . N \geq k-1$.

If $d=k$, then note that since $h^{0}\left(C,\left.\mathcal{O}_{S}(N)\right|_{C}\right) \geq 2$, then $N . C \geq k+\operatorname{length}(\xi)$, or else we get a contradiction on the gonality of $C$. But this gives us $N . C=N^{2}+M . N=2+M . N \geq$ $k+k-M . N$, yielding $M . N \geq k-1$.

Special case 3. In the case where $N^{2}=4$ with $\phi(N)=2$, we have by assumption that $N . C-2 \geq \mu(L) \geq k+1$. Since $N . C=M . N+N^{2}=N . M+4$, it follows that $M . N \geq k-1$.

General case. Now suppose $M \geq N, N^{2} \geq 4$ and $(M-N)^{2}>0$. If $N^{2}=4$, we suppose that $\phi(N) \neq 2$. We first show that there exists an elliptic curve $E$ such that the conditions of Lemma 3.5 are satisfied.

By Lemma 3.2, $M-N \equiv a_{1} E_{1}+\cdots+a_{m} E_{m}$ for some elliptic curves $E_{i}$ satisfying $E_{i} . E_{j}>0$ for $i \neq j$, and integers $a_{i}>0$. Since $(M-N)^{2}>0$, we must have $m \geq 2$, and so $(M-N) . E>0$ for any elliptic curve $E$.

Applying Lemma 3.2 again, we see that there exist positive integers $b_{i}$ and elliptic curves $E_{i}^{\prime}$ satisfying $1 \leq E_{i}^{\prime} . E_{j}^{\prime} \leq 2$ for $i \neq j$, such that $N \equiv b_{1} E_{1}^{\prime}+\cdots+b_{m^{\prime}} E_{m^{\prime}}^{\prime}$. This implies that $N-\left(b_{1} E_{1}^{\prime}+\cdots+b_{m^{\prime}} E_{m^{\prime}}^{\prime}\right)$ is linearly equivalent to either 0 or $K_{S}$. If $h^{0}\left(S, \mathcal{O}_{S}(N-E)\right)=1$, where $E<N$, the only way this can happen is that $N-E$ is linearly equivalent to a stationary elliptic curve or a sum of two elliptic curves $E_{1}^{\prime \prime}, E_{2}^{\prime \prime}$ satisfying $2 E_{1}^{\prime \prime} \sim 2 E_{2}^{\prime \prime}$. But the first case implies $N^{2} \leq 4$ with $\phi(N)=2$, which is a contradiction; and in the second case, $N \sim E+E_{1}^{\prime \prime}+E_{2}^{\prime \prime}$ such that $h^{0}\left(S, \mathcal{O}_{S}\left(N-E_{i}^{\prime \prime}\right)\right) \geq 2$ for $i=1,2$, so that Lemma 3.5 can still be applied.

It follows that there exists an elliptic curve $E$ such that $\left.(M+E)\right|_{C}$ contributes to $\operatorname{Cliff}(C)$. By [KL13, Corollary 1.2], the general curve $C$ in $|L|$ has Clifford index $k-2$. Recalling from 
the proof of Lemma 3.5 that $H^{0}\left(S, \mathcal{O}_{S}(M+E)\right) \hookrightarrow H^{0}\left(C, \mathcal{O}_{C}(M+E)\right)$, we get

$$
\begin{aligned}
k-2 & =\operatorname{Cliff}(C) \\
& \leq \operatorname{Cliff}\left(\left.(M+E)\right|_{C}\right)=(M+E) \cdot C-2\left(h^{0}\left(C, \mathcal{O}_{C}(M+E)\right)-1\right) \\
& \leq M \cdot(M+N)+E \cdot C-(M+E)^{2} \\
& =M \cdot N+E . C-2 M . E \\
& =M \cdot N+E \cdot M+E \cdot N-2 M . E \\
& =M \cdot N-E \cdot(M-N) \\
& \leq M \cdot N-1,
\end{aligned}
$$

as desired.

The two following lemmas are used in the proof of Proposition 3.9. The first one gives a parameter space for the extensions of $\mathcal{O}_{S}(M)$ and $\mathcal{O}_{S}(N) \otimes \mathcal{I}_{\xi}$, and is the second place in this article where we use the assumption that $L$ is ample. The second lemma is important when we count the dimensions of possible pairs $(C, A)$ that can arise from the same vector-bundle $\mathcal{E}$.

Lemma 3.7. Suppose $\mathcal{O}_{S}(M)$ and $\mathcal{O}_{S}(N)$ are two line-bundles on $S$ satisfying M.L $\geq$ N.L, and let $\xi$ be a non-empty zero-dimensional subscheme on $S$ of length $\ell>0$. Then all isomorphism-classes of extensions of $\mathcal{O}_{S}(M)$ and $\mathcal{O}_{S}(N) \otimes \mathcal{I}_{\xi}$ are parametrised by

$$
\mathbb{P} \operatorname{Ext}^{1}\left(\mathcal{O}_{S}(N) \otimes \mathcal{I}_{\xi}, \mathcal{O}_{S}(M)\right) \cong \mathbb{P} H^{1}\left(S, \mathcal{O}_{S}\left(N-M+K_{S}\right) \otimes \mathcal{I}_{\xi}\right)^{\vee},
$$

which has dimension

$$
\ell+h^{1}\left(S, \mathcal{O}_{S}(M-N)\right)-h^{2}\left(S, \mathcal{O}_{S}(M-N)\right)-1
$$

Proof. The isomorphism classes of extensions of $\mathcal{O}_{S}(M)$ and $\mathcal{O}_{S}(N) \otimes \mathcal{I}_{\xi}$ are parametrised by $\mathbb{P} \operatorname{Ext}^{1}\left(\mathcal{O}_{S}(N) \otimes \mathcal{I}_{\xi}, \mathcal{O}_{S}(M)\right) \cong \mathbb{P} H^{1}\left(S, \mathcal{O}_{S}\left(N-M+K_{S}\right) \otimes \mathcal{I}_{\xi}\right)^{\vee}$, by [Fri98, pages 36 and 39].

To find an expression for $h^{1}\left(S, \mathcal{O}_{S}\left(N-M+K_{S}\right) \otimes \mathcal{I}_{\xi}\right)$, we tensor the exact sequence

$$
0 \rightarrow \mathcal{I}_{\xi} \rightarrow \mathcal{O}_{S} \rightarrow \mathcal{O}_{\xi} \rightarrow 0
$$

with $\mathcal{O}_{S}\left(N-M+K_{S}\right)$ and take global sections, yielding

$$
\begin{aligned}
0 \rightarrow H^{0}\left(S, \mathcal{O}_{S}(N-\right. & \left.\left.M+K_{S}\right) \otimes \mathcal{I}_{\xi}\right) \rightarrow H^{0}\left(S, \mathcal{O}_{S}\left(N-M+K_{S}\right)\right) \rightarrow \mathbb{C}^{\ell} \\
& \rightarrow H^{1}\left(S, \mathcal{O}_{S}\left(N-M+K_{S}\right) \otimes \mathcal{I}_{\xi}\right) \rightarrow H^{1}\left(S, \mathcal{O}_{S}\left(N-M+K_{S}\right)\right) \rightarrow 0 .
\end{aligned}
$$

We have $h^{0}\left(S, \mathcal{O}_{S}\left(N-M+K_{S}\right) \otimes \mathcal{I}_{\xi}\right)=0$ because $\left(N-M+K_{S}\right) . L \leq 0$ by assumption, and using that $L$ is ample together with the Nakai-Moishezon criterion.

The result now follows by Serre duality.

Lemma 3.8. Suppose $\mathcal{E}$ is an extension of $\mathcal{O}_{S}(M)$ and $\mathcal{O}_{S}(N) \otimes \mathcal{I}_{\xi}$ so that we have a sequence as in Proposition 3.3. Then $h^{0}\left(S, \mathcal{E} \otimes \mathcal{E}^{\vee}\right) \geq h^{0}\left(S, \mathcal{O}_{S}(M-N)\right)$.

Proof. If $M \ngtr N$ or $M \sim N+K_{S}$, we have $h^{0}\left(S, \mathcal{O}_{S}(M-N)\right) \leq 1$, so there is nothing to prove. So assume that $M \gtrless N$.

Tensor the sequence

$$
0 \rightarrow \mathcal{O}_{S}(M) \rightarrow \mathcal{E} \rightarrow \mathcal{O}_{S}(N) \otimes \mathcal{I}_{\xi} \rightarrow 0
$$


by $\mathcal{E}^{\vee}$. Taking global sections, we see that $h^{0}\left(S, \mathcal{E} \otimes \mathcal{E}^{\vee}\right) \geq h^{0}\left(S, \mathcal{E}^{\vee} \otimes \mathcal{O}_{S}(M)\right)$. By Serre duality, we have $h^{0}\left(S, \mathcal{E}^{\vee} \otimes \mathcal{O}_{S}(M)\right)=h^{2}\left(S, \mathcal{E} \otimes \mathcal{O}_{S}\left(-M+K_{S}\right)\right)$. It thus suffices to prove that $h^{2}\left(S, \mathcal{E} \otimes \mathcal{O}_{S}\left(-M+K_{S}\right)\right) \geq h^{0}\left(S, \mathcal{O}_{S}(M-N)\right)$.

Tensor (20) with $\mathcal{O}_{S}\left(-M+K_{S}\right)$. Taking cohomology, we get

$$
H^{2}\left(S, \mathcal{E} \otimes \mathcal{O}_{S}\left(-M+K_{S}\right)\right) \rightarrow H^{2}\left(S, \mathcal{O}_{S}\left(N-M+K_{S}\right) \otimes \mathcal{I}_{\xi}\right) \rightarrow 0 .
$$

So we have $h^{2}\left(S, \mathcal{E} \otimes \mathcal{O}_{S}\left(-M+K_{S}\right)\right) \geq h^{2}\left(S, \mathcal{O}_{S}\left(N-M+K_{S}\right) \otimes \mathcal{I}_{\xi}\right)$.

But if we consider

$$
0 \rightarrow \mathcal{I}_{\xi} \rightarrow \mathcal{O}_{S} \rightarrow \mathcal{O}_{\xi} \rightarrow 0
$$

tensored with $\mathcal{O}_{S}\left(N-M+K_{S}\right)$ and take cohomology, we see that $h^{2}\left(S, \mathcal{O}_{S}\left(N-M+K_{S}\right) \otimes\right.$ $\left.\mathcal{I}_{\xi}\right)=h^{2}\left(S, \mathcal{O}_{S}\left(N-M+K_{S}\right)\right)$, which by Serre duality equals $h^{0}\left(S, \mathcal{O}_{S}(M-N)\right)$. The result follows.

We are now ready to state and prove the main result of this section.

Proposition 3.9. Suppose that for general $(C, A)$ in $\mathcal{W}$, the vector-bundles $\mathcal{E}_{C, A}$ are non- $\mu_{L^{-}}$ stable, and suppose that $\mu(L)>2 \phi(L)=k$. Then $\operatorname{dim} \mathcal{W} \leq g-1+d-k$.

Proof. By assumption, for general $(C, A)$ in $\mathcal{W}, \mathcal{E}_{C, A}$ sits inside an exact sequence as in Proposition (3.3). We prove the proposition by making a parameter-count of all pairs $(C, A)$ such that $\mathcal{E}_{C, A}$ is non- $\mu_{L^{-}}$-stable, making a similar construction as the one done in AF11, Section 3] in the case of non-simple vector-bundles on K3-surfaces.

We divide this proof into three cases. We first consider the case where the vector-bundles $\mathcal{E}_{C, A}$ are indecomposable with $\ell>0$, followed by the indecomposable case when $\ell=0$. Finally, we consider the case where the $\mathcal{E}_{C, A}$ 's are decomposable.

The case where the general $\mathcal{E}_{C, A}$ 's are indecomposable with $\ell>0$. Fix a line-bundle $\mathcal{O}_{S}(N)$ such that $|N|$ is base-component free, and which satisfies the following conditions: $(L-N) . L \geq N . L, d \geq(L-N) . N$, and $d-(L-N) . N \leq h^{0}\left(S, \mathcal{O}_{S}(N)\right)$. Set $M:=L-N$ and $\ell:=d-M . N$. Note that these conditions imply that $h^{1}\left(S, \mathcal{O}_{S}(M)\right)=0, h^{0}\left(S, \mathcal{O}_{S}(M)\right) \geq 2$ and $h^{2}\left(S, \mathcal{O}_{S}(M)\right)=0$.

Let $\tilde{\mathcal{P}}_{N, \ell}$ be the family of vector-bundles that are extensions of $\mathcal{O}_{S}(M)$ and $\mathcal{O}_{S}(N) \otimes \mathcal{I}_{\xi}$ where $\xi$ is a zero-dimensional subscheme of length $\ell$. For $0 \leq i \leq 2$ (see Lemma 3.4), define

$$
\mathcal{P}_{N, \ell, i}:=\left\{[\mathcal{E}] \in \tilde{\mathcal{P}}_{N, \ell} \mid h^{2}(S, \mathcal{E})=0, h^{1}(S, \mathcal{E})=i,\right.
$$

and $\mathcal{E}$ is globally generated away from a finite set of points\}.

We can think of $\mathcal{P}_{N, \ell, i}$ as extensions of $\mathcal{O}_{S}(M)$ and $\mathcal{O}_{S}(N) \otimes \mathcal{I}_{\xi}$ where $\xi$ imposes $\ell-i$ conditions on $|N|$. Note that this puts restrictions on the dimensions of possible $\xi$ 's that can be considered. Whereas the Hilbert scheme $S^{[\ell]}$ parametrises all possible $\xi$ 's of length $\ell$, the $\xi$ 's that impose $\ell-i$ conditions on $|N|$ can be found by considering elements $\eta$ of $S^{[\ell-i]}$ and add base-points of $\left|\mathcal{O}_{S}(N) \otimes \mathcal{I}_{\eta}\right|$. Since $\mathcal{E}$ is globally generated away from a finite set of points, then $\left|\mathcal{O}_{S}(N) \otimes \mathcal{I}_{\xi}\right|$ is base-component free, and so there are only a finite set of base-points in $\left|\mathcal{O}_{S}(N) \otimes \mathcal{I}_{\eta}\right|$.

(*) It follows that there are at most $2 \ell-2 i$ dimensions of $\xi^{\prime}$ s in $S^{[\ell]}$ that impose $\ell-i$ conditions on $|N|$.

Still following the construction of [AF11, Section3], we let $\mathcal{G}_{N, \ell, i}$ be the Grassmann bundle over $\mathcal{P}_{N, \ell, i}$ classifying pairs $([\mathcal{E}], \Lambda)$ with $[\mathcal{E}] \in \mathcal{P}_{N, \ell, i}$ and $\Lambda \in \operatorname{Gr}\left(2, h^{0}(S, \mathcal{E})\right)$. (Note that $h^{0}(S, \mathcal{E})=h^{0}\left(S, \mathcal{O}_{S}(M)\right)+h^{0}\left(S, \mathcal{O}_{S}(N)\right)-\ell+i$, and is thus constant.) 
By assumption, we have a rational map

$$
h_{N, \ell, i}: \mathcal{G}_{N, \ell, i} \rightarrow \mathcal{W}_{d}^{1}(|L|)
$$

given by $h_{N, \ell, i}([\mathcal{E}], \Lambda):=\left(C_{\Lambda}, A_{\Lambda}\right)$ (see sequence (12)). The dimension of each fibre of $h_{N, \ell, i}$ is found by finding the dimension of all surjections $\mathcal{E} \rightarrow \mathcal{O}_{C}\left(K_{C_{\Lambda}}-A_{\Lambda}+\left.K_{S}\right|_{C_{\Lambda}}\right)$ and subtract the dimension of all morphisms from $\mathcal{O}_{C}\left(K_{D_{\Lambda}}-A_{\Lambda}+\left.K_{S}\right|_{C_{\Lambda}}\right)$ to itself (which is 1).

By tensoring (12) with $\mathcal{E}^{\vee}$ and taking global sections, we see that $h^{0}\left(C, \mathcal{E}^{\vee} \otimes \mathcal{O}_{C}\left(K_{C_{\Lambda}}-\right.\right.$ $\left.\left.A_{\Lambda}+\left.K_{S}\right|_{C_{\Lambda}}\right)\right)=h^{0}\left(S, \mathcal{E} \otimes \mathcal{E}^{\vee}\right)$. Since the general morphism from $\mathcal{E}$ to $\mathcal{O}_{C}\left(K_{C_{\Lambda}}-A_{\Lambda}+\left.K_{S}\right|_{C_{\Lambda}}\right)$ is surjective, it follows that the dimension of each fibre of $h_{N, \ell, i}$ is equal to $h^{0}\left(S, \mathcal{E} \otimes \mathcal{E}^{\vee}\right)-1$. By Lemma 3.8, this is $\geq h^{0}\left(S, \mathcal{O}_{S}(M-N)\right)-1$.

Letting $e$ be $h^{0}(S, \mathcal{E})$ for any vector-bundle $\mathcal{E}$ in $\mathcal{P}_{N, \ell, i}$, we conclude that $\operatorname{dim} \mathcal{W}_{d}^{1}|L|$ is bounded by $\operatorname{dim} \mathcal{P}_{N, \ell, i}+\operatorname{dim} \operatorname{Gr}(2, e)-h^{0}\left(S, \mathcal{O}_{S}(M-N)\right)+1$.

By $(*)$ combined with Lemma 3.7, and using that $\ell=d-M . N$, we have $\operatorname{dim} \mathcal{P}_{N, \ell, i} \leq$ $2 \ell-2 i+\ell+h^{1}\left(S, \mathcal{O}_{S}(M-N)\right)-h^{2}\left(S, \mathcal{O}_{S}(M-N)\right)-1=3 d-3 M . N-2 i+h^{1}\left(S, \mathcal{O}_{S}(M-\right.$ $N))-h^{2}\left(S, \mathcal{O}_{S}(M-N)\right)-1$. We furthermore have $\operatorname{dim} \operatorname{Gr}(2, e)=2(e-2)=2 e-4=$ $2 \chi(S, \mathcal{E})+2 i-4=2(g+1-d)+2 i-4=2 g-2 d+2 i-2$.

This gives us in total

$$
\begin{aligned}
\operatorname{dim} \mathcal{W} \leq 3 d-3 M . N-2 i-\chi\left(S, \mathcal{O}_{S}(M-N)\right)-1+2 g-2 d+2 i-1 \\
=2 g-3 M . N+d-2-\chi\left(S, \mathcal{O}_{S}(M+N)\right)+2 M . N \\
\quad=2 g-M . N+d-2-g=g-2+d-M . N .
\end{aligned}
$$

By Proposition 3.6, $M . N \geq k-1$, and it follows that

$$
\operatorname{dim} \mathcal{W} \leq g-1+d-k .
$$

The case where the general $\mathcal{E}_{C, A}$ 's are indecomposable with $\ell=0$. In this case, we also construct the same family $\mathcal{P}_{N, \ell, i}=\mathcal{P}_{N, 0, i}$ of vector-bundles as in the previous case. By Fri98, all extensions of $\mathcal{O}_{S}(M)$ and $\mathcal{O}_{S}(N)$ are parametrised by $\mathbb{P} H^{1}\left(S, \mathcal{O}_{S}\left(N-M+K_{S}\right)\right)^{\vee}=$ $\mathbb{P} H^{1}\left(S, \mathcal{O}_{S}(M-N)\right)$. As in the previous case, we consider the same family of vector-bundles $\mathcal{P}_{N, 0, i}$, where $i \leq 2$, together with the grassmannian bundle $\mathcal{G}_{N, 0, i}$. This gives us the bound

$$
\operatorname{dim} \mathcal{W}_{d}^{1}|L| \leq h^{1}\left(S, \mathcal{O}_{S}(M-N)\right)-1+\operatorname{dim} \operatorname{Gr}(2, e)-h^{0}\left(S, \mathcal{O}_{S}(M-N)\right)+1,
$$

where $e=h^{0}(S, \mathcal{E})$ for the extensions $\mathcal{E}$ with $h^{1}(S, \mathcal{E})=i$.

Since there are no indecomposable extensions of $\mathcal{O}_{S}(M)$ and $\mathcal{O}_{S}(N)$ when $M \sim N+K_{S}$ (since then $\left.h^{1}\left(S, \mathcal{O}_{S}(M-N)\right)=0\right)$, we can by Proposition 3.3 (a) assume that $h^{2}\left(S, \mathcal{O}_{S}(M-\right.$ $N))=0$. We have, as before, $\operatorname{dim} \operatorname{Gr}(2, e)=2 g-2 d+2 i-2$, and $\chi\left(\mathcal{O}_{S}(M-N)\right)=g-2 M . N$.

Note that since $\ell=d-M . N$, we have $d=M . N$ in this case. It follows that $\operatorname{dim} \mathcal{W}_{d}^{1}|L| \leq-\chi\left(\mathcal{O}_{S}(M-N)\right)+2 g-2 d+2 i-2+1=-g+2 M . N+2 g-2 d+2 i-1=g-1+2 i$.

Now, if $i=0$, we are done. So suppose $i>0$. Since $h^{1}\left(S, \mathcal{O}_{S}(M)\right)=0$ (by Proposition 3.3), it follows that $h^{1}\left(S, \mathcal{O}_{S}(N)\right)=i$, and so by Theorem 3.1, $N^{2}=0$. But then, $N . C=N . M=d$, and since $\left.N\right|_{C} \geq A$ (by Proposition 3.3), it follows that these vector-bundles only yield one single $g_{d}^{1}$ for each curve $C$.

The case where the general $\mathcal{E}_{C, A}$ 's are decomposable. Now suppose $\mathcal{E}_{C, A}$ is decomposable for general $(A, C)$. In that case, we must have $\ell=0$, and so $M \cdot N=d$. Note also that there can only be finitely many different $\mathcal{E}_{C, A}$ in this case, and so we will here show that the 
image of the map $f_{\mathcal{E}}: \operatorname{Gr}\left(h^{0}(S, \mathcal{E}), 2\right) \rightarrow \mathcal{W}_{d}^{1}|L|$ is of dimension at most $g-1$, thus implying that $\operatorname{dim} W_{d}^{1}(C)=0$ (given the assumptions in the proposition).

As argued in the indecomposable case, we have

$$
\operatorname{dimim} f_{\mathcal{E}}=2\left(h^{0}(S, \mathcal{E})-2\right)-h^{0}\left(S, \mathcal{E} \otimes \mathcal{E}^{\vee}\right)+1 .
$$

Since $\mathcal{E}$ is decomposable, we have $\mathcal{E} \otimes \mathcal{E}^{\vee} \cong \mathcal{O}_{S}^{\oplus 2} \oplus \mathcal{O}_{S}(M-N) \oplus \mathcal{O}_{S}(N-M)$.

By Proposition 3.3. we have either $M \sim N+K_{S}$ or $h^{2}\left(S, \mathcal{O}_{S}(M-N)\right)=0$.

If $M \sim N+K_{S}$, then both $M^{2}>0$ and $N^{2}>0$, and so $h^{1}(S, \mathcal{E})=h^{1}\left(S, \mathcal{O}_{S}(M)\right)+$ $h^{1}\left(S, \mathcal{O}_{S}(N)\right)=0$, by Theorem 3.1. In this case, we have $h^{0}(S, \mathcal{E})=g-d+1$ and $d=M . N=$ $\frac{g-1}{2}$, and so

$$
\operatorname{dimim} f_{\mathcal{E}}=2 g-2 \cdot \frac{g-1}{2}-3-h^{0}(M-N)-h^{0}(N-M) \leq g-2 .
$$

If $h^{2}\left(S, \mathcal{O}_{S}(M-N)\right)=0$, then let $i=h^{1}(S, \mathcal{E})$. As in the case where the $\mathcal{E}$ 's are indecomposable with $\ell=0$, we also here get $N^{2}=0$ if $i>0$, and hence that $\left.N\right|_{C}=A$. We thus get one single $g_{d}^{1}$ for each curve $C$.

Now suppose $i=0$. This implies that $h^{0}(S, \mathcal{E})=g-d+1$, and so

$$
\operatorname{dimim} f_{\mathcal{E}} \leq 2(g-d-1)-h^{0}\left(S, \mathcal{O}_{S}(M-N)\right)-1 .
$$

Using Riemann-Roch together with the assumption that $h^{2}\left(S, \mathcal{O}_{S}(M-N)\right)=0$, we get $h^{0}\left(S, \mathcal{O}_{S}(M-N)\right) \geq \frac{1}{2} C^{2}-2 M . N+1=g-2 M . N=g-2 d$, which gives us

$$
\operatorname{dimim} f_{\mathcal{E}} \leq g-3 \text {. }
$$

\section{The Case where the $\mathcal{E}_{C, A}$ 'S ARE $\mu_{L}$-Stable}

In this section, we cover the cases where $\mathcal{E}_{C, A}$ is $\mu_{L}$-stable for general $(C, A)$ in $\mathcal{W}$. It is here not possible to do a parameter count in order to obtain a suitable bound, but we prove here instead that $\operatorname{dim} \operatorname{ker}\left(\mu_{0, A}\right) \leq 2$, yielding that $\operatorname{dim} W_{d}^{1}(C)=d-k$ for $d \leq g-k$ for the curves in question.

Note that, by Assumption (17), we cannot have $h^{0}\left(C, \mathcal{O}_{C}\left(A+\left.K_{S}\right|_{C}\right)\right) \geq 3$ for general $(C, A)$ in $\mathcal{W}$, since otherwise, by subtracting points, we would have more than $\operatorname{dim} W_{d}^{1}(C)$ dimensions of $g_{d-1}^{1}$ 's, which is impossible. In the following propositions, we therefore only need to consider the cases where $h^{0}\left(C, \mathcal{O}_{C}\left(A+\left.K_{S}\right|_{C}\right)\right)=2$ or $\leq 1$, respectively.

Proposition 4.1. Suppose that $\mathcal{E}_{C, A}$ is $\mu_{L}$-stable, and that $h^{0}\left(C, \mathcal{O}_{C}\left(A+\left.K_{S}\right|_{C}\right)\right)=2$, for general $(C, A)$ in $\mathcal{W}$. Then $\operatorname{dim} \mathcal{W} \leq g-1+d-k$.

Proof. If $h^{0}\left(C, \mathcal{O}_{C}\left(A+\left.K_{S}\right|_{C}\right)\right)=2$ for the general pairs $(C, A)$ in $\mathcal{W}$, then by (5) , we can assume that $\mathcal{O}_{C}\left(A+\left.K_{S}\right|_{C}\right)$ is also base-point free for general $A$, and so these pairs $(C, A+$ $\left.\left.K_{S}\right|_{C}\right)$ define vector-bundles $\mathcal{E}_{C, A+\left.K_{S}\right|_{C}}$. If these vector-bundles are non- $\mu_{L}$-stable for general $(C, A)$ in $\mathcal{W}$, then by Proposition [3.9, we get at most $g-1+d-k$ dimensions of pairs $\left(C, A+\left.K_{S}\right|_{C}\right)$, and so there must also be at most that many dimensions of pairs $(C, A)$. So suppose the vector-bundles are $\mu_{L}$-stable.

The vector-bundles $\mathcal{E}_{C, A+\left.K_{S}\right|_{C}}$ lie inside a sequence

$$
0 \rightarrow H^{0}\left(S, \mathcal{O}_{S}\left(A+K_{S} \mid C\right)\right)^{\vee} \otimes \mathcal{O}_{S} \rightarrow \mathcal{E}_{C, A+\left.K_{S}\right|_{C}} \rightarrow \mathcal{O}_{C}\left(K_{C}-A\right) \rightarrow 0 .
$$


Now, tensoring this sequence with $\mathcal{E}_{C, A}^{\vee}$ and taking global sections, we get

$$
\begin{aligned}
0 \rightarrow H^{0}\left(S, \mathcal{E}_{C, A}^{\vee}\right)^{\oplus 2} \rightarrow H^{0}\left(S, \mathcal{E}_{C, A+\left.K_{S}\right|_{C}} \otimes \mathcal{E}_{C, A}^{\vee}\right) \rightarrow H^{0}\left(C, \mathcal{O}_{C}\left(K_{C}-A\right)\right. & \left.\otimes \mathcal{E}_{C, A}^{\vee}\right) \\
& \rightarrow H^{1}\left(S, \mathcal{E}_{C, A}^{\vee}\right)^{\oplus 2} .
\end{aligned}
$$

Since $h^{0}\left(S, \mathcal{E}_{C, A}^{\vee}\right)=h^{1}\left(S, \mathcal{E}_{C, A}^{\vee}\right)=0$, then by Proposition [2.2, $h^{0}\left(S, \mathcal{E}_{C, A+\left.K_{S}\right|_{C}} \otimes \mathcal{E}_{C, A}^{\vee}\right)=$ $\operatorname{dim} \operatorname{ker} \mu_{0, A}$.

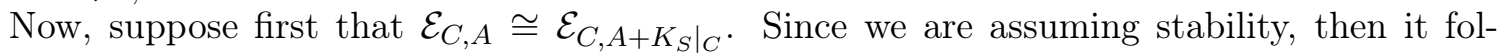
lows that the vector-bundles are simple, and so $h^{0}\left(S, \mathcal{E}_{C, A} \otimes \mathscr{E}_{C, A}\right)=1$, and it follows that $\operatorname{dim} \operatorname{ker} \mu_{0, A}=1$. By (1), $\operatorname{dim} W_{d}^{1}(C)=-g+2 d-1$, and by putting $d \leq g-k$, we have $\operatorname{dim} W_{d}^{1}(C) \leq d-k-1$.

Now assume that $\mathcal{E}_{C, A} ¥ \mathcal{E}_{C, A+\left.K_{S}\right|_{C}}$. Since both $\mathcal{E}_{C, A}$ and $\mathcal{E}_{C, A+\left.K_{S}\right|_{C}}$ are $\mu_{L}$-stable, then (noting that $\mu_{L}\left(\mathcal{E}_{C, A}\right)=\mu_{L}\left(\mathcal{E}_{C, A+\left.K_{S}\right|_{C}}\right)$ ), we have $h^{0}\left(S, \mathcal{E}_{C, A+\left.K_{S}\right|_{C}} \otimes \mathcal{E}_{C, A}^{\vee}\right)=0$, and so $\operatorname{dim} \operatorname{ker} \mu_{0, A}=0$, and $\operatorname{dim} W_{d}^{1}(C)=d-k$ by (1) and (4).

Proposition 4.2. Suppose that $\mathcal{E}_{C, A}$ is $\mu_{L}$-stable, and that $h^{0}\left(C, \mathcal{O}_{S}\left(A+\left.K_{S}\right|_{C}\right)\right) \leq 1$, for general $(C, A)$ in $\mathcal{W}$. Then $\operatorname{dim} \operatorname{ker} \mu_{0, A} \leq 2$. It follows that if $d \leq g-k$, then $\operatorname{dim} \mathcal{W} \leq$ $g-1+d-k$.

Proof. Tensoring the sequence (7) by $\mathcal{E}_{C, A}^{\vee} \otimes \mathcal{O}_{S}\left(K_{S}\right)$ and taking cohomology, one gets

$$
\begin{array}{r}
0 \rightarrow H^{0}\left(S,\left(\mathcal{E}_{C, A}^{\vee} \otimes \mathcal{O}_{S}\left(K_{S}\right)\right)\right)^{\oplus 2} \rightarrow H^{0}\left(S, \mathcal{E}_{C, A} \otimes \mathscr{E}_{C, A}^{\vee} \otimes \mathcal{O}_{S}\left(K_{S}\right)\right) \rightarrow H^{0}\left(C, \mathcal{E}_{C, A}^{\vee} \otimes \mathcal{O}_{C}\left(K_{C}-A\right)\right) \\
\rightarrow H^{1}\left(S,\left(\mathcal{E}_{C, A}^{\vee} \otimes \mathcal{O}_{S}\left(K_{S}\right)\right)^{\oplus 2}\right) .
\end{array}
$$

We have $H^{0}\left(S, \mathcal{E}_{C, A} \otimes \mathscr{E}_{C, A}^{\vee} \otimes \mathcal{O}_{S}\left(K_{S}\right)\right)=0$ by the stableness assumption, and so it follows that $H^{0}\left(C, \mathcal{E}_{C, A}^{\vee} \otimes \mathcal{O}_{C}\left(K_{C}-A\right)\right)$ injects into $H^{1}\left(S,\left(\mathcal{E}_{C, A}^{\vee} \otimes \mathcal{O}_{S}\left(K_{S}\right)\right)^{\oplus 2}\right)$.

Since $h^{1}\left(S, \mathcal{E}_{C, A}^{\vee} \otimes \mathcal{O}_{S}\left(K_{S}\right)\right)=h^{1}\left(S, \mathcal{E}_{C, A}\right)=h^{0}\left(C, \mathcal{O}_{C}\left(A+\left.K_{S}\right|_{C}\right)\right.$ ) (by (11)), which by assumption is $\leq 1$, we have $h^{1}\left(C, \mathcal{E}_{C, A}^{\vee} \otimes \mathcal{O}_{C}\left(K_{C}-A\right)\right) \leq 2$. By Proposition 2.2, dim ker $\mu_{0, A} \leq$ 2 .

By (11), it follows that if $\operatorname{dim} \operatorname{ker} \mu_{0, A} \leq 2$ for $[A]$ general in a component $W$ of $W_{d}^{1}(C)$, then $\operatorname{dim} W=-g+2 d$. By putting $d \leq g-k$, the result follows.

Remark 4.3. It is interesting to note that the same result can be obtained by considering the moduli-space $M$ of $\mu_{L}$-stable vector-bundles of rank 2 with $c_{1}=L$ and $c_{2}=d$ on $S$. It is known (see e.g. [Kim06, Remark, page 768]) that the dimension of the tangent space at $\mathcal{E}$ is given by

$$
\operatorname{dim} T_{\mathcal{E}} M=4 c_{2}-c_{1}^{2}-3+h^{2}\left(S, \mathcal{E} \otimes \mathcal{E}^{\vee}\right) .
$$

Since $h^{2}\left(S, \mathcal{E} \otimes \mathcal{E}^{\vee}\right)=0$ in our case, it follows that the dimension is given by $4 d-L^{2}-3=$ $4 d-2 g-1$.

By considering all possible injections $\Lambda \hookrightarrow H^{0}(S, \mathcal{E})$, as done in the proof of Proposition 3.9. we obtain $\operatorname{dim} \mathcal{W} \leq 2 d-1$ using this approach, or equivalenty, $\operatorname{dim} W_{d}^{1}(C) \leq 2 d-g$. We have $2 d-g \leq d-k$ precisely when $d \leq g-k$.

Proof of Theorem [1.1. Suppose that $\pi: \mathcal{W} \rightarrow|L|$ dominates. From (15), we can assume that for general $(C, A) \in \mathcal{W}$, we have that $|A|$ is base-point free. We can therefore for these $(C, A)$ consider vector-bundles $\mathcal{E}_{C, A}$. 
If for general $(C, A)$ in $\mathcal{W}$ we have $\mathcal{E}_{C, A}$ non- $\mu_{L}$-stable, then by Proposition 3.9, we have $\operatorname{dim} \mathcal{W} \leq g-1+d-k$. If for general $(C, A)$ in $\mathcal{W}$ we have $\mathcal{E}_{C, A} \mu_{L^{-}}$stable, then we have the same bound by Propositions 4.1 and 4.2 .

Since $\operatorname{dim}|L|=g-1$, the result follows.

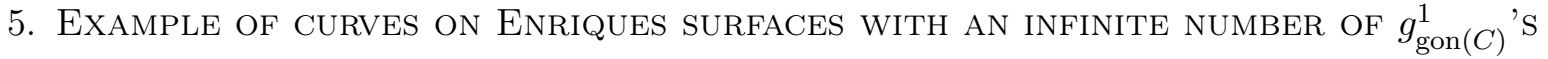

We here present an example of curves with an infinite number of $g_{\operatorname{gon}(C)}^{1}$ 's.

Example 5.1. Let $S$ be any Enriques surface (which is possibly nodal). Let $L=n\left(E_{1}+E_{2}\right)$ for $n \geq 3$, where $E_{1} \cdot E_{2}=2$, in which case, $k=\mu(L)$ by [KL09, Corollary 1.5 (a)]. Then there exists a sub-linear system $\mathfrak{d} \subseteq|L|$ of smooth curves such that for general $C \in \mathfrak{d}$, there exist infinitely many $g_{\text {gon }(C)}^{1}$ 's.

Indeed, let $B=\mathcal{O}_{S}\left(E_{1}+E_{2}\right)$, consider the map $f_{B}: S \rightarrow \mathbb{P}^{2}$, and let $\mathfrak{d}=f^{*}\left|\mathcal{O}_{\mathbb{P}^{2}}(n)\right|$. This is then a sub-linear system of $|L|$, consisting of all curves that map 4-1 onto curves of $\left|\mathcal{O}_{\mathbb{P}^{2}}(n)\right|$. By Bertini's theorem, since this linear system is base-point free, the generic elements are smooth.

One constructs infinitely many $g_{B . L-4}^{1}$ 's on a generic smooth curve $C \in \mathfrak{d}$ in the following way: Let $C=f^{-1}\left(C^{\prime}\right)$, where $C^{\prime}$ is smooth in $\mathcal{O}_{\mathbb{P}^{2}}(n)$. Then $C$ is also smooth. We let the $g_{B . L-4}^{1}$ 's be $\left.f\right|_{C} ^{*}\left(\mathcal{O}_{C^{\prime}}(1) \otimes \mathcal{O}_{C^{\prime}}(-P)\right)$, where $P$ is any point on $C^{\prime}$. (On $C$, this is the same as subtracting one point $Q$ on $\left.B\right|_{C}$ and noting that $|B|_{C}-Q \mid$ has three base-points $f^{-1}(f(Q))-Q$ that can also be subtracted.)

By [KL09, Corollary 1.6], the minimal gonality is always at most 2 less than the generic gonality, and the generic gonality is given by $B . L-2$ by [KL09, Corollary 1.5], so in our case, it follows that $\operatorname{gon}(C)=B . L-4$. Since $n \geq 3$, we are ensured that the $g_{B . L-4}^{1}$ 's are distinct.

These $g_{B . L-4}^{1}$ 's are, as far as we know, new examples of curves $C$ with infinitely many $g_{\mathrm{gon}(C)}^{1}$ 's. The curves are furthermore non-exceptional.

These curves $C$ are $4-1$ coverings of plane curves, and the $g_{\text {gon }(C)}^{1}$ 's are induced from the $g_{\text {gon }\left(C^{\prime}\right)}^{1}$ 's. According to the Castelnuovo-Severi inequality (see e.g. [Kan84]), whenever we have an $m-1$ covering from a curve $C$ to a curve $C^{\prime}$, if $g(C)>m g\left(C^{\prime}\right)+(m-1)(d-1)$, then any base-point free $g_{d}^{1}$ on $C$ is induced by a base-point free linear system on $C^{\prime}$. In particular, if $d=\operatorname{gon}(C)$ and $C^{\prime}$ has infitely many $g_{\text {gon }\left(C^{\prime}\right)}^{1}$ 's, then $C$ also has infinitely many $g_{\operatorname{gon}(C)}^{1}$ 's. However, in this example, $g(C) \leq m g\left(C^{\prime}\right)+(m-1)(d-1)$.

Furthermore, by CM91, Corollary 2.3.1], any exceptional curve $C$ has infinitely many $g_{\text {gon }(C)}^{1}$ 's. However, by [KL13, Theorem 1.1], the only exceptional curves $C$ on Enriques surfaces are isomorphic to smooth plane quintics and satisfy $C^{2}=10$. It follows that the curves in our example are non-exceptional.

\section{REFERENCES}

[ACGH85] A. Arbarello, M. Cornalba, P.A. Griffiths, and J. Harris, Geometry of algebraic curves, vol. 1, Springer Verlag, 1985.

[AF11] M. Aprodu and G. Farkas, Green's conjecture for curves on arbitrary K3 surfaces, Compositio Math 147 (2011), 839-851.

[Apr05] M. Aprodu, Remarks on syzygies of d-gonal curves, Math. Res. Lett. 12 (2005), 387-400.

[CM91] M. Coppens and G. Martens, Secant spaces and Clifford's theorem, Compositio Mathematica 78 (1991), 193-212. 
[CP95] C. Ciliberto and G. Pareschi, Pencils of minimal degree on curves on a K3 surface, Journal für die reine und angewandte Mathematik 460 (1995), 14-36.

[Fri98] R. Friedman, Algebraic surfaces and holomorphic vector bundles, Springer Verlag, 1998.

[GH80] P. Griffiths and J. Harris, The dimension of the variety of special linear systems on a general curve, Duke Math. J 47 (1980), 233-272.

[GL87] M. Green and R. Lazarsfeld, Special divisors on curves on a K3 surface, Inventiones mathematicae 89 (1987), no. 2, 357-370.

[Gre84] M. Green, Koszul cohomology and the geometry of projective varieties (appendix by M. Green and R. Lazarsfeld), Journal of differential geometry 6 (1984), 125-171.

[Kan84] E. Kani, On Castelnuovo's equivalence defect, Journal für die reine und angewandte Mathematik 352 (1984), 24-70.

[Kem71] G. Kempf, Schubert methods with an application to algebraic curves, Stichting Mathematisch Centrum, 1971.

[Kim06] H. Kim, Stable vector bundles of rank two on Enriques surfaces, J. Korean Math. Soc 43 (2006), no. 4, 765-782.

[KL72] S.L. Kleiman and D. Laksov, On the existence of special divisors, American Journal of Mathematics 94 (1972), no. 2, 431-436.

[KL07] A.L. Knutsen and A.F. Lopez, A sharp vanishing theorem for line bundles on K3 or Enriques surfaces, Proceedings of the American Mathematical Society 135 (2007), no. 11, 3495-3498.

[KL09] Brill-Noether theory for curves on Enriques surfaces, I: the positive cone and gonality, Mathematische Zeitschrift 261 (2009), 659-690.

[KL13] _ Brill-Noether theory of curves on Enriques surfaces, II. The Clifford index, arXiv preprint arXiv:1308.1074 (2013).

[Knu01] A.L. Knutsen, On kth-order embeddings of K3 surfaces and Enriques surfaces, manuscripta mathematica 104 (2001), no. 2, 211-237.

[Knu03] Gonality and Clifford index of curves on K3 surfaces, Archiv der Mathematik 80 (2003), no. 3, 235-238.

[Knu09a] On secant spaces to Enriques surfaces, Bulletin of the Belgian Mathematical Society-Simon Stevin 16 (2009), no. 5, 907-931.

[Knu09b] - On two conjectures for curves on K3 surfaces, International Journal of Mathematics 20 (2009), 1547-1560.

[Laz86] R. Lazarsfeld, Brill-Noether-Petri without degenerations, J. Diff. Geom. 23 (1986), 299-307.

[LC12] M. Lelli-Chiesa, Green's conjecture for curves on rational surfaces with an anticanonical pencil, Mathematische Zeitschrift (2012), 1-12.

[Mar68] H.H. Martens, On the varieties of special divisors on a curve. II, Journal für die reine und angewandte Mathematik (Crelles Journal) 1968 (1968), no. 233, 89-100.

[Mar84] G. Martens, On dimension theorems of the varieties of special divisors on a curve, Mathematische Annalen 267 (1984), no. 2, 279-288.

[Par95] G. Pareschi, A proof of Lazarsfeld's theorem on curves on K3 surfaces, J. Alg. Geom. 4 (1995), 195-200.

[Tyu87] A.N. Tyurin, Cycles, curves and vector bundles on an algebraic surface, Duke Math. J 54 (1987), no. $1,1-26$.

Nils Henry Rasmussen and Shengtian Zhou

nils.h.rasmussen@hit.no, shengtian.zhou@hit.no

Telemark University College, Dept. of Teacher Education

Lærerskolevegen 40, 3679 NOTODDEN, Norway 